\title{
Biofilm Quantification in Listeria monocytogenes 4B Serotype isolated from Animals of Gujarat State, India
}

\author{
Mahendra Mohan Yadav ${ }^{1,2 *}$ \\ ${ }^{1}$ Department of Veterinary Microbiology, College of Veterinary Science E Animal Husbandry, Anand, Gujarat, INDIA \\ ${ }^{2}$ Department of Animal Husbandry \& Dairy Science, RCSM College of Agriculture, Kolhapur, Maharashtra, INDIA
}

"Corresponding author: MM Yadav; E-mail:drmahendrayadav@rediffmail.com

Received: 07 Nov., 2020

Revised: 01 Jan., 2021

Accepted: 06 Jan., 2021

\begin{abstract}
The purpose of this study was to assess slime production and quantification of biofilm in a set of well-characterized Listeria monocytogenes strains isolated from healthy and clinically affected animal. In all 28 strains of $L$. monocytogenes belonging to serotype $4 \mathrm{~b}$ having proved in vitro pathogenicity potential is included in the study. Slime production was determined by cultivation of the organisms on Congo red agar medium, while quantification of biofilm was performed with the help of microtitre plate assay. Out of 28 isolates, $22(78.57 \%)$ strains of L. monocytogenes produced slime. The rest of the $6(21.43 \%)$ isolates were negative for slime production. For biofilm production, out of the 28 strains, 5 (17.86\%), 18 (64.29\%), and 5 (17.86 $\%$ ) were found moderate, weak and negative, respectively. Strains belonging to Listeria monocytogenes serotype $4 \mathrm{~b}$ showed inconsistent results regarding biofilm production. Biofilm productivity exhibited profound intra-strain variations irrespective of source of isolation. As L. monocytogenes are biofilm producers, this increases the probability of occurrence of animal and human infection. Further, as L. monocytogenes produces biofilm, infections caused by this bacterium may be underestimated because diagnoses in the presence of biofilm are difficult.
\end{abstract}

\section{HIGHLIGHTS}

(0 Listeria monocytogenes serotype $4 \mathrm{~b}$ produces slime that may increase the occurrence of animal and human infections.

(0 Listeria monocytogenes serotype $4 \mathrm{~b}$ showed inconsistency in biofilm production.

Keywords: Animals, Listeria monocytogenes, biofilm

Listeriosis is one of the important bacterial diseases of animals and a zoonosis with a broad distribution; it has considerable economic and public health impact. Listeria monocytogenes, a facultative intracellular pathogen responsible for Listeriosis, has been isolated from healthy and diseased animals, birds and human beings. It is a wellknown cause of abortion, encephalitis and septicemia both in animals and human beings. Since the bacterium is ubiquitous in nature, found in soil and vegetation, it is easily contracted and transmitted to animals and contaminate process of food production (Matle et al., 2020). In particular, the psychrotropic nature of organism allows replication in refrigerated condition, ready-to-eat food products that have been contaminated during processing and packaging, resulting in food-borne outbreaks that are characterized by widespread distribution and relatively high mortality rates.

When organized as biofilm, the self-produced extracellular polymeric matrix gives extra protection to bacteria from harsh environmental conditions such as desiccation, nutrient deprivation, or disinfectant treatment (Bridier et al., 2011; Esbelin et al., 2018). Within a biofilm, bacteria become attached to a surface where they exist in complex communities that are able to interact with each other

How to cite this article: Yadav, M.M. (2021). Biofilm quantification in Listeria monocytogenes $4 \mathrm{~b}$ serotype isolated from animals of Gujarat state, India. J. Anim. Res., 11(1): 51-57.

Source of Support: None; Conflict of Interest: None 
through intracellular communication and thus rapidly adapt to changing environments. Biofilm formed in animal environments was of special significance as it has the potential to act as the chronic source of microorganism that may lead to transmission of diseases. Moreover, exposure to a stress factor can provide cross-adaptation to subsequent exposure to other stresses (Bergholz et al., 2012). Complex mechanisms regulate bacterial sessile growth and biofilm formation represented by adhesion, maturation and dispersal steps, each affected by intrinsic and extrinsic factors (Matle et al., 2020).

Perusal of literature suggest that there is scanty of research about role of bacterial biofilms in animals especially under Indian conditions, but they are believed to be involved in many diseases such as pneumonia, liver abscesses, enteritis, wound infections and mastitis infections (Melchior et al., 2006a,b). These infections can be caused by environmental organisms, such as $P$. aeruginosa, Staphylococcus aureus etc. that are commonly found in wound infections, as well as by species of bacteria that constitute part of the normal microflora of animals. Through a combination of endogenous and exogenous factors these generally harmless commensals may become pathogenic. And considering extent and rate of bacterial attachment, it has been seen that microorganisms get attached to more rapidly to hydrophobic and nonpolar surfaces as Teflon and other plastics rather than to glass and other materials having hydrophilic properties (Choudhary et al., 2020). These plastics are nowadays frequently used in dairy industry under Indian conditions. Biofilm formation in microtiter plates is certainly the most commonly used method to grow and study biofilm. This simple design is very popular due to its high-throughput screening capacities, low cost, and easy handling (Thibeaux et al., 2020). The microtitre plate has the advantage of analyzing adhesion of multiple bacterial strains or growth conditions within each experiment. So, present study was carried out in a set of well-characterized L. monocytogenes strains for assessing biofilm detection and quantitation with the help of microtitre plate assay. The strains included clinical and animal sources.

\section{MATERIALS AND METHODS}

\section{Ethical approval}

As per the Committee for the Purpose of Control and Supervision of Experiments on Animals guidelines, a study involving collection of clinical samples under field conditions does not require approval of Institute Animal Ethics Committee. Blood samples were collected by licensed veterinarians as per standard sample collection methods without any harm or stress to the animals.

\section{Procurement of standard bacterial culture}

The standard strain of L. monocytogenes 4b (MTCC 1143) used in the study was obtained from the Microbial Type Culture Collection and Gene Bank, Institute of Microbial Technology, Chandigarh, India. The well-characterized strains of L. monocytogenes isolated from different health status of animals and sources are shown in Table 1 and Table 2. All the 28 strains are maintained in Department of Veterinary Microbiology, College of Veterinary Science \& Animal Husbandry, Anand (India).

Table 1: Health status of animals from which L. monocytogenes strains were isolated

\begin{tabular}{llll}
\hline Sl. No. & Source of Isolation & L. monocytogenes \\
\hline 1 & Apparently healthy Sheep & Faecal Samples & 7 \\
2 & Cattle and Buffalo with reproductive tract disorders & Deep vaginal Swabs & 9 \\
& & Faecal Samples & 2 \\
3 & Lactating Animals & Milk Samples & 4 \\
& & Faecal Samples & 1 \\
4 & Clinical Mastitis & Feed Samples & 1 \\
5 & Apparently healthy Zoo Animals & Mastitic Milk & 3 \\
\hline
\end{tabular}


Table 2: Isolation of L. monocytogenes from Animals' Sources

\begin{tabular}{llllll}
\hline Isolate No. & $\begin{array}{l}\text { Animal source of } \\
\text { Isolation }\end{array}$ & Source of Sample & Isolate No. & $\begin{array}{l}\text { Animal source of } \\
\text { Isolation }\end{array}$ & Source of Sample \\
\hline LM1 & Sheep & Feces & LM15 & Cattle & Vaginal Swab \\
LM2 & Sheep & Feces & LM16 & Cattle & Vaginal Swab \\
LM3 & Sheep & Feces & LM17 & Cattle & Vaginal Swab \\
LM4 & Sheep & Feces & LM18 & Cattle & Vaginal Swab \\
LM5 & Sheep & Feces & LM19 & Cattle & Feces \\
LM6 & Sheep & Feces & LM20 & Cattle & Milk \\
LM7 & Sheep & Feces & LM21 & Cattle & Milk \\
LM8 & Buffalo & Feces & LM22 & Bufffalo & Milk \\
LM9 & Buffalo & Feces & LM23 & Cattle & Milk \\
LM10 & Buffalo & Vaginal Swab & LM24 & Environment & Feed \\
LM11 & Buffalo & Vaginal Swab & LM25 & Cattle & Milk \\
LM12 & Buffalo & Vaginal Swab & LM26 & Cattle & Milk \\
LM13 & Buffalo & Vaginal Swab & LM27 & Cattle & Milk \\
LM14 & Buffalo & Vaginal Swab & LM28 & Zoo animal & Feces \\
\hline
\end{tabular}

\section{Characterization of $L$. monocytogenes}

All the 28 strains were characterized for haemolysis activity, Christie Atkins Munch Peterson (CAMP) test (Annonymous, 1994), Phosphatidylinositol-specific Phospholipase C (PI-PLC) assay (Leclercq, 2004), Phosphatidylcholine-Specific Phospholipase C (PC-PLC) assay (Coffey et al., 1996). All the strains were found positive for aforementioned characters.

All the 28 strains were serotyped as L. monocytogenes $4 \mathrm{~b}$ using multiplex PCR assay following the methodology as described by Doumith et al. (2004) with suitable modifications.

\section{Slime production assay}

Slime production of the isolates was determined by cultivation of the organisms on Congo red agar medium as per the method described by Freeman et al. (1989). Each strain was streaked on the Congo red agar medium and incubated aerobically at $37^{\circ} \mathrm{C}$ for $24 \mathrm{~h}$ followed by further incubation at room temperature $\left(25^{\circ} \mathrm{C}\right)$ for $48 \mathrm{~h}$. The production of rough black colonies by the strains indicated production of slime.

\section{Quantification of biofilm}

Quantification of biofilm production in plastic microtitreplate was based on the previously described method of Stepanovic et al. (2004) with slight modification as follows. The wells of a sterile 96-well flat-bottomed polystyrene microtitreplate (Laxbro Ltd. India) were used for the test. The test organisms were grown in congo-red broth at $37{ }^{\circ} \mathrm{C}$ for $18 \mathrm{~h}$ incubation. The microtitreplate were filled with $230 \mu \mathrm{l}$ of the congo-red broth. A quantity of $20 \mu \mathrm{l}$ of overnight bacterial culture of the test isolates was added into each well. Each isolates was tested in triplicate. The negative control wells contained broth only. The plates were incubated aerobically for $24 \mathrm{~h}$ at $35^{\circ} \mathrm{C}$. The content of the plate was then poured off and the wells washed three times with $300 \mu \mathrm{l}$ of sterile distilled water. The remaining attached bacteria were fixed with $250 \mu \mathrm{l}$ of methanol per well, and after 15 min microtitre plates were emptied and air-dried. The microtitre plates were stained with $250 \mu 1$ per well of Crystal violet used for Gram staining for $5 \mathrm{~min}$. Excess stain was rinsed off by placing the microtitre plates under running tap water. After the microtitre plates were air dried, the dye bound to the adherent cells was resolubilized with $250 \mu \mathrm{l}$ of $33 \%$ (v/v) glacial acetic acid per well. The optical density (O.D.) of each well was measured at $570 \mathrm{~nm}$ using an automated Multiscan EX reader (Thermo Electron Corporation Ltd, Navi Mumbai, India).

Mean O.D of the test isolates tested in triplicates was taken as final O.D of the test isolates. Based on the O.D. 
produced by bacterial films, strains were classified into the following categories: no biofilm producers, weak, moderate or strong biofilm producers, as described by Stepanovic et al. (2004). Briefly, the cut-off O.D. (O.D.c) was defined as three standard deviations above the mean O.D. of the negative control. Strains were classified as follows: O.D. $<$ O.D.c $=$ no biofilm producer, O.D.c < O.D. $<(2 \times$ O.D.c $)=$ weak biofilm producer, $(2 \times$ O.D.c $)$ $<$ O.D. $<(4 \times$ O.D.c $)=$ moderate biofilm producer and $(4$ $\times$ O.D.c $)<$ O.D. $=$ strong biofilm producer.

\section{RESULTS AND DISCUSSION}

The objective of this study was to detect slime and biofilm formation from strains that were characterized has having pathogenic potential by in vitro pathogenicity testing. The term slime has been used to characterize biofilm formation phenotypically on CRA medium.

\section{Slime production}

The isolates were studied for detection of slime production on Congo-red agar medium (CRA). Formation of rough black colonies on CRA plates was considered to be indicative of slime production compared to the red smooth colonies of non-slime producing strains. All the $28 \mathrm{~L}$. monocytogenes isolates were streaked on Congo-red agar in order to detect slime production. Out of 28 isolates, 22 $(78.57 \%)$ strains of L. monocytogenes produced slime. The rest of the $6(21.43 \%)$ strains were negative for slime production (Table 3). Probably this was the first report on detection of slime in L. monocytogenes under Indian conditions. The production of slime, an extracellular substance which surrounds multiple cell layers, facilitates bacterial adherence. Slime production was investigated as a possible major determinant of bacterial adherence to biotic and abiotic surfaces. Slime production in Staphylococcus

Table 3: Biofilm and Slime Production of L. monocytogenes Isolates

\begin{tabular}{|c|c|c|c|}
\hline \multirow{2}{*}{ Isolate No. } & \multirow{2}{*}{ Slime production } & \multicolumn{2}{|c|}{ Biofilm production } \\
\hline & & O.D & Remark \\
\hline LM 1 & + & 0.287 & Moderate \\
\hline LM 2 & + & 0.144 & Weak \\
\hline LM 3 & + & 0.387 & Moderate \\
\hline LM 4 & + & 0.248 & Weak \\
\hline LM 5 & + & 0.198 & Weak \\
\hline LM 6 & + & 0.200 & Weak \\
\hline LM 7 & + & 0.147 & Weak \\
\hline LM 8 & + & 0.166 & Weak \\
\hline LM 9 & + & 0.184 & Weak \\
\hline LM 10 & - & 0.137 & Negative \\
\hline LM 11 & + & 0.147 & Weak \\
\hline LM 12 & + & 0.192 & Weak \\
\hline LM 13 & + & 0.209 & Weak \\
\hline LM 14 & + & 0.210 & Weak \\
\hline LM 15 & + & 0.173 & Weak \\
\hline LM 16 & + & 0.143 & Weak \\
\hline LM 17 & + & 0.389 & Moderate \\
\hline LM 18 & - & 0.127 & Negative \\
\hline LM 19 & + & 0.378 & Moderate \\
\hline LM 20 & - & 0.133 & Negative \\
\hline LM 21 & + & 0.141 & Weak \\
\hline LM 22 & + & 0.259 & Weak \\
\hline LM 23 & + & 0.290 & Weak \\
\hline LM 24 & + & 0.401 & Moderate \\
\hline LM 25 & - & 0.128 & Negative \\
\hline LM 26 & - & 0.134 & Negative \\
\hline LM 27 & - & 0.144 & Weak \\
\hline LM 28 & + & 0.174 & Weak \\
\hline
\end{tabular}


spp. has been postulated to be associated with their innate resistance to phagocytosis, adhesion, micro colony formation and antibiotic resistance. It has been used as a marker to indicate the ability of the organisms like Staphylococcus aureus to adhere the tissues in diseases like mastitis (Vasudevan et al., 2003).

\section{Quantification of biofilm}

The individual strains of $L$. monocytogenes varied in their biofilm forming ability. Out of total 28, $23(82.14 \%)$ isolates were found to produce biofilm whereas $5(17.86 \%)$ were found negative for biofilm. Biofilm production of $L$. monocytogenes isolates by microtitre plate assay showed O.D. values in the range of 0.128 to 0.438 . The cut off $O$. D. was 0.140 . The O.D. value of isolates less than 0.140 was considered negative, while that of 0.141 to 0.280 as weak, 0.281 to 0.560 as moderate and more than 0.560 as strong biofilm producer. The O.D values of all the 28 strains were shown in Table 3. Out of the 28 strains, 5 $(17.86 \%), 18(64.29 \%)$, and $5(17.86 \%)$ were found moderate, weak and negative, respectively, for biofilm production. The strains that produced moderate biofilm belong feces of sheep (2), vaginal swab taken from cattle having reproductive disorder (1), feces of lactating cattle (1), feed (1). Eighteen strains that were classified as weak biofilm producer belong to feces of sheep (5), feces of buffalo (2), vaginal swab taken from buffalo having reproductive disorder (4), vaginal swab taken from cattle having reproductive disorder (2), milk samples of cattle (1) and buffalo (1), while 5 strains that where negative for biofilm production were vaginal swab taken from buffalo having reproductive disorder (1), vaginal swab taken from cattle having reproductive disorder (1), milk samples of cattle (1) and clinical mastitis milk sample taken from cattle (2). Previous study of Doijad et al. (2015) also showed that none of the strain from animal clinical cases, human clinical cases, and meat exhibited strong biofilm formation. Biofilm productivity exhibited profound inter-strain variations depending on growth conditions that resulted in inconsistent associations between biofilm phenotype and serotypes throughout the different conditions Lee $e t$ al. (2019). Further investigations on genes of unknown function as well as a time-course omics approaches such as transcriptomics and proteomics will help decipher the complex mechanisms of biofilm formation
Harveya et al. (2007) reported that out 127 of 138 strains $(92.0 \%)$ were classified as weak, 9 of 138 strains $(6.5 \%)$ as moderate and only 2 of 138 strains $(1.5 \%)$ as strong biofilm formers. The strains included environmental, animal, food (persistent and sporadic strains) and clinical isolates. The present findings were in agreement with the above report. Though, the terms slime and biofilm are used interchangeably, the expression of the slime production cannot be correlated with the production of biofilm. As it can be observed in the present study that non-slime producing isolates were also found to be biofilm producers. This finding is in agreement with that of Vasudevan et al. (2003) where it was found that three slime negative strains of Staphylococcus aureus were al so biofilm producers. Therefore, microtiter plate assay can be more consistent assay for biofilm formation in comparison to that of slime production. It has been suggested that bacteria in response to changing environmental conditions were able to switch between a free-living, virulent state and a surface attached, less virulent state. Kalmokoff et al.'s (2001) finding that only 1 of 36 clinical L. monocytogenes strains formed biofilm on stainless steel surfaces support this suggestion. Bacteria in biofilms were generally more resistant to environmental stresses than their planktonic counterparts. The biofilm forming capability of the Listeria spps. makes them particularly successful in colonizing surfaces within the host thus being responsible for persistence infections (Kamelia et al., 2016). Furthermore, studies will also be necessary to understand the mechanisms underlying different rates of biofilm growth among strains of $L$. monocytogenes. Knowledge gained in these areas will be an important step towards prevention of biofilms and elimination of persistent strains from food processing environments.

Presence of persistent strains of L. monocytogenes in the farm or/and milk line could be due to the residence of mastitic cows and/or a dwelling biofilm in milking machinery and utensils Latorre et al., 2013). Biofilms has been linked with bovine mastitis (Melchior et al., $2006 a, b)$. Various organisms of veterinary importance have been successfully grown as biofilms using a Calgary Biofilm Device (CBD) including Corynebacterium pseudotuberculosis, E. coli, P. aeruginosa, Staphylococcus hyicus and $S$. aureus. But in present study, it is clear that strains belonging to serotype $4 \mathrm{~b}$ (predominant serotype responsible for the animal listeriosis and Listeria 
associated food borne outbreaks and considered to be the most virulent serotype, having proven in vitro virulence associated characteristics), showed inconsistent results regarding biofilm production, and none of them was strong biofilm producer. Osman et al. (2016) also recorded that $4 \mathrm{~b}$ strains did not exhibit strong biofilm formation that could have a drastic outcome in the dairy industry with a consequent hazardous implication on food safety. Though epidemiological evidence points to biofilms as a source of several infectious diseases, the exact mechanisms by which biofilm-associated microorganisms elicit disease are poorly understood (Donlan and Costerton, 2002). L. monocytogenes biofilm formation is probably controlled by a complex regulation network involved in variable genes required for the different biological pathways. PrfA, a key transcriptional activator that regulates most of the known listerial virulence gene expression, has been shown to promote L. monocytogenes biofilm formation. Loss of PrfA dramatically altered gene expression patterns in $L$. monocytogenes biofilm and resulted in reduced ability of the biofilm formation (Luo et al., 2013).

\section{CONCLUSION}

The present investigation was aimed to assess slime production and quantitation of biofilm production in a set of well-characterized L. monocytogenes strains isolated from healthy and clinicaly affected animal sources. Strains belonging to serotype $4 \mathrm{~b}$ showed inconsistent results regarding biofilm production, and also none of them were strong biofilm producer. Biofilm productivity exhibited profound intra-strain variations irrespective of source of isolation. Biofilm formation is a complex process regulated by diverse factors, and further studies will be necessary to understand the mechanisms underlying the different rates of biofilm production among the strains of L. monocytogenes, which will support prevention of biofilms and eliminating persistent strains from animal and animal based food environment. Biofilm formation by such pathogens in the zoonotic pathogen is a matter of concern not only to Veterinarians but also to the human health.

\section{ACKNOWLEDGEMENTS}

I thank the Dean/Principal, College of Veterinary Science and Animal Husbandry, Anand, Gujarat, India for providing necessary facilities to carry out the research work.

\section{REFERENCES}

An, Y.H. and Friedman, R.J. 2000. Handbook of bacterial adhesion: principles, methods, and applications. Humana Press, Totowa, N.J.

Annonymous, 1994. Bureau of Indian Standards. Microbiology general guidance for the detection of Listeria monocytogenes. Committee Draft, CD, 11290.

Bergholz, T.M., Bowen, B., Wiedmann, M. and Boor, K.J. 2012. Listeria monocytogenes shows temperature-dependent and -independent responses to salt stress, including responses that induce cross-protection against other stresses. Appl. Environ. Microbiol., 78: 2602-2612.

Bridier, A., Briandet, R., Thomas, V. and Dubois-Brissonnet, F. 2011. Resistance of bacterial biofilms to disinfectants: a review. Biofouling, 27, 1017-1032.

Choudhary, P., Singh, S. and Agarwal, V. 2020. Microbial Biofilms. IntechOpen. A Book Chapter, http://dx.doi. org/10.5772/intechopen.90790, pp 1-14.

Coffey, A., Rombouts, F.M. and Abee, T. 1996. Influence of environmental parameters on phosphatidylcholine phospholipase $\mathrm{C}$ production in Listeria monocytogenes: a convenient method to differentiate L. monocytogenes from other Listeria species. Appl. Environ. Microbiol., 62: 1252 1256.

Doijad, S.P., Barbuddhe, S.B., Garg, S., Poharkar, K.V., Kalorey, D.R., Kurkure, N.V., Rawool, D.B. and Chakraborty, T. 2015. Biofilm- forming abilities of Listeria monocytogenes serotypes isolated from different sources. PLoS One, 10: 1371-1374.

Donlan, R. M. 2002. Biofilms: microbial life on surfaces. Emerg. Infect. Dis., 8: 881-890.

Donlan, R.M. and Costerton, J.W. 2002. Biofilms: Survival Mechanisms of Clinically Relevant Microorganisms. Clin. Microbiol. Rev., 15: 167-193.

Doumith, M., Buchrieser, C., Glaser, P., Jacquet, C. and Martin P. 2004. Differentiation of the Major Listeria monocytogenes Serovars by Multiplex PCR. J. Clin. Microbiol., 42: 3819 3822 .

Esbelin, J., Santos, T. and Hébraud, M. 2018. Desiccation: an environmental and food industry stress that bacteria commonly face. Food Microbiol., 69: 82-88

Freeman, D.J., Falkner, F.R. and Keane, C.T. 1989.: New method for detecting slime production by coagulase negative Staphylococci. J. Clin. Pathol., 4: 872-874. 
Harveya, J., Keenana, K.P. and Gilmour, A. 2007. Assessing biofilm formation by Listeria monocytogenes strains. Food Microbiol., 24: 380-392.

Kalmokoff, M.L., Austin, J.W., Wan, X.D., Sanders, G., Banerjee, S. and Farber, G. 2001. Adsorption, attachment and biofilm formation among isolates of Listeria monocytogenes using model conditions. J. Appl. Microbiol., 91: 725-734.

Kamelia, M.O., Ahmed, S., Usama, H.A., Essam, H.M., Ahmed, O. and Zolnikov, T. 2016. Determination of virulence and antibiotic resistance pattern of biofilm producing Listeria species isolated from retail raw milk. BMC Microbiol., 16: 263.

Latorre, A.A., Van Kessel, J.A.S., Karns, J.S., Zurakowski, M.J., Pradhan, A.K., Boor, K.J., Adolph, E., Sukhnanand, S. and Schukken, Y.H. 2011. Increased in vitro adherence and on-farm persistence of predominant and persistent Listeria monocytogenes strains in the milking system. Appl. Environ. Microbiol., 77:3 676-84.

Leclercq, A. 2004. Atypical colonial morphology and low recoveries of Listeria monocytogenes strains on Oxford, PALCAM, Rapid'L.mono and ALOA solid media. $J$. Microbiol. Methods., 57: 251-258.

Lee, B.H., Cole, S., Badel-Berchoux, S., Guillierm, L., Felix, B., Krezdorn, N., Hébraud, M., Bernardi, T., Sultan, I. and Piveteau, P. 2019. Biofilm Formation of Listeria monocytogenes Strains Under Food Processing Environments and Pan-Genome-Wide Association Study. Frontiers in Microbiol., 10: 2698-2715.

Luo, Q., Shang, J., Feng, X., Guo, X., Zhang, L. and Zhou, Q. 2013. PrfA Led to Reduced Biofilm Formation and Contributed to Altered Gene Expression Patterns in BiofilmForming Listeria monocytogenes. Curr. Microbiol., 67: 372378.
Matle, I., Mbatha, K.R. and Madoroba, E. 2020. A review of Listeria monocytogenes from meat and meat products: Epidemiology, virulence factors, antimicrobial resistance and diagnosis. Onderstepoort J. Vet. Res., 87(1): a1869.

Melchior MB, Fink-Gremmels J. and Gaastra W. 2006a. Comparative assessment of the antimicrobial susceptibility of Staphylococcus aureus isolates from bovine mastitis in biofilm versus planktonic culture. J. Vet. Med. B Infect. Dis. Vet. Pub. Health, 53: 326-332.

Melchior, M.B., Vaarkamp, H. and Fink-Gremmels, J. 2006b. Biofilms: a role in recurrent mastitis infections? Vet. J., 171: 398-407.

Osman, K.M., Samir, A., Abo-Shama, U.H., Mohamed, E.H., Orabi, A. and Zolnikov, T. 2016. Determination of virulence and antibiotic resistance pattern of biofilm producing Listeria species isolated from retail raw milk. BMC Microbiol., 16: 263-275

Stepanovic, S., Cirkovic, I., Ranin, L. and Svabic-Vlahovic, M. 2004. Biofilm formation by Salmonella spp. and Listeria monocytogenes on plastic surface. Letters Appl. Microbiol., 38: $428-432$.

Thibeaux R., Kainiu M. and Goarant C. 2020. Biofilm Formation and Quantification Using the 96-Microtiter Plate. In: Koizumi N., Picardeau M. (eds) Leptospira spp.. Methods in Molecular Biology, vol 2134. Humana, New York.

Vasudevan Nair, M.K.M., Annamalai, T. and Venkatanarayanan, K. S. 2003. Phenotypic and genotypic characterization of bovine mastitis isolates of Staphylococcus aureus in biofilm formation. Vet. Microbiol., 92: 179-185. 
